\title{
Ethnic and Gender Influences on Adaptive Behaviour of Some Nigerian Children
}

\author{
Esther Foluke Akinsola (Ph.D.) \\ Department of Psychology \\ University of Lagos, Lagos, Nigeria \\ Ruth Goma \\ Department of Psychology \\ University of Lagos, Lagos, Nigeria
}

\begin{abstract}
In this manuscript we summarize the results of a pilot study carried out to field test a newly developed adaptive behaviour scale for children that is culturally sensitive and expected to be culturally appropriate for Nigerian children. In addition we attempted to determine whether adaptive behaviour skill competence increased with age and the roles played by ethnicity and gender in the acquisition and demonstration of adaptive behaviour skills. The children were sampled from the three Nigerian major ethnic groups namely Yoruba, Igbo, and Hausa who are resident in Lagos, Nigeria. 282 children aged 2-9 years were rated by their class teachers using the newly developed adaptive behaviour scale for children [3]. The results obtained from this study indicated that: (i) the development and demonstration of adaptive behaviour skill competence increased with age; (ii) at particular age group levels, ethnicity significantly and differentially increased adaptive behaviour skill competence in all the skill domains; (iii) in the communication domain and also at particular age group levels gender significantly and differentially increased adaptive behaviour skill competence of the children. There was no significant main effect for ethnicity and gender. The scale items were found to be culturally appropriate for Nigerian children. It was therefore concluded that further research is needed to establish an overall influence of ethnicity and gender on the acquisition and demonstration of adaptive behaviour skill competence in Nigerian children.
\end{abstract}

Key words: Ethnic, Gender, Adaptive behaviour, Nigerian Children

\section{INTRODUCTION}

According to [13], adaptation is a psychobiological construct that refers to the individual's active modifications to cope effectively with the demands of the natural, social, and designed environments. [5] as reported by [13] stated that adaptations encompass domestic activities and interpersonal relationships. According to [9], as reported by [13] the origins of motor, cognitive, affective and social adaptive competencies lie in early childhood and that such adaptations are reflections of maturation interacting with formal or incidental learning. Through the interaction between maturation and formal or incidental learning, all adaptations relate to their context of development and improve with practice and application. From this perspective, cultural and environmental factors are implicated in the development and expression of adaptive behaviour.

Adaptive behaviour is defined as the performance of the daily activities required for personal and social sufficiency. It is the collection of conceptual, social, and practical skills that have been learned by people for them to function in their everyday lives [2]. 
Conceptual skills include receptive and expressive language, reading and writing, and self direction. Social skills embrace responsibility, obeying rules and laws, and competence in interpersonal interactions. Practical skills include personal, and instrumental self-care activities such as toileting, dressing properly, eating, occupational skills, and moneymanagement skills [10].

Three principles govern adaptive behaviour. First, adaptive behaviour is age related and environmentally based. Its assessment is perceived as an important method of assessing development of individuals across age and cultural groups, because expectations may differ depending on age and within different settings and contexts [10]. It is thus expected that competency and complexity in adaptive behaviour would increase with age and may be improved through education. Second, adaptive behaviour is defined by expectations and standards of other people and its adequacy is judged by people who live, work, and interact with the individual. Third, adaptive behaviour is measured by typical performance and not ability. Although ability is necessary for performance of daily activities, adaptive behaviour will be inadequate if the ability is not demonstrated when it is required [11]. In addition to age, ethnicity and gender may make some contributions to the variance in adaptive behaviour scores. [10] reviewed some studies on race/ethnic and socioeconomic group as well as gender differences in adaptive behaviour. They reported that $[6,7]$ found that ethnicity was related to parent and teacher ratings of children's adaptive behaviour as measured by the Adaptive Behaviour Inventory for children and Teacher's Questionnaire. They reported that [8] and [4] found small ethnic differences in the school version of the Adaptive Behaviour Scale, and that [12], reported similar scores by ethnicity on the survey form of the Vineland Adaptive Behaviour Scale after controlling for parental education level and age. For gender differences, [10], reported that $[6,7]$ found gender to be related to parent and teacher ratings of children's scores on the Adaptive Behaviour Inventory for children and Teacher's Questionnaire. They also reported that [8], and [4] found that gender contributed to scores on the school version of the Adaptive Behaviour Scale; and that [12] reported similar scores for males and females on the Survey Form of the Vineland Adaptive Behaviour Scales (2nd edition). A more recent study on cultural and ethnic differences in children's adaptive behaviour ratings reported by mothers was conducted by [13]. They studied the adaptive behaviour of 2-5yr old children from Italian and Austrian/German Italian ethnic groups using the Vinland Adaptive Behaviour Scale. They found that Austrian/German Italian children were reported to be more verbally expressive than Italian children, have better Daily Living Skills including personal care, domestic tasks and community responsibilities. Both ethnic groups were reported to be similar in motor skills. Furthermore, it was reported that Austrian/German Italian mothers rated their children higher in Socialization domain including interpersonal relationships, play skills, and coping skills. The ethnic differences were explained in terms of Italian acculturation orientation which was found to be significantly different in the two ethnic groups. These differences reflect differential environmental adaptive demands that mould the adaptive behaviour skills of the children

[13]. It was therefore expected that differences in the adaptive behaviour scores of the children in this study would reflect variations in the environmental adaptive demands between the ethnic groups. Lagos (study location), being a metropolitan city and a former capital of Nigeria, it contains samples from all ethnic groups in Nigeria including Yoruba, Igbo and Hausa which are the three major ethnic groups. The study sample was drawn from these three ethnic groups. 


\section{Rational for Study:}

The Yoruba, Igbo and Hausa ethnic groups have different traditional life styles which are expected to impact on their child rearing practices.

The Yoruba people are largely farmers or traders who live in large cities. Their traditional culture is hinged on oral tradition. They use proverbs, songs, and modelling to teach their children and their families societal values and appropriate behaviour in their communities. Majority of them are native speakers of Yoruba language. Emphasis in their adaptive behaviour training for their children would reflect their life style and survival values. Their children stay with them and they teach them adaptation and survival skills, such as learning through selfeffort, cooperation, and modelling and working hard [1].

The Igbo people live in smaller independent villages and towns with elected councils and council heads. They are mostly craftsmen, farmers and traders with high level of business sense. They are also native speakers of their dialects and they also have their own way of teaching their children their values and appropriate behaviour.

The Hausa people also live in small towns and villages or indigenous cities. They grow crops, raise life stock like cattle and engage in small scale industry. Due to the fact that raising of life stock like cattle is one of the main occupations of the Hausas many of them are nomads that migrate from one vegetation area to another area which may be many kilometres apart. As a result, they do not have permanent settlements, and are more of first language speakers than second language speakers. They too have their own way of teaching their children adaptive behaviour skills. The differences in the lifestyles of these groups are expected to influence their child rearing practices and consequently the development and the expression of adaptive behaviour skills in their children

\section{Purpose of Research}

The main purpose of the present study was to determine if the development and mastery of adaptive behaviour skills of the children sampled would increase as their ages increase and whether the mastery of the skills would vary across ethnic groups and gender. It was hypothesized that (i)Acquisition of and Competence in adaptive behaviour skills would increase with age; (ii) Children from Yoruba ethnic group would demonstrate higher levels of competence in adaptive behaviour skills than children from Igbo and Hausa ethnic groups; (iii) Female children would demonstrate higher levels of adaptive behaviour skill competence than male children.

\section{Participants:}

\section{METHOD}

282 children with their ages ranging from 2-9 years and made up of 137 males and 145 females participated in the study. The sample consisted of 178 Yoruba children, 93 Igbo children and 11 Hausa children. They were selected from crèche, nursery and primary sections of three schools located in the mainland area of Lagos metropolis in Nigeria.

\section{Instrument:}

The school edition of the newly developed culturally sensitive adaptive behaviour scale modelled after Vineland Adaptive Behaviour Scale and Adaptive Behaviour Assessment System was used to collect the data. This study was a pilot one to field test the instrument to determine the appropriateness of the test items and its environmental appropriateness. 
The scale consists of 60 items covering four areas of adaptive behaviour skills namely Communication, Daily Living, Socialisation, and Motor skills. Each section has 15 items and the items are rated on a three-point scale of $1-3$, with $1=$ always, $2=$ often, and $3=$ seldom. 1 attracts a score of 3, 2 a score of 2 and 3 a score of one. The maximum score for each section is 45 and the minimum score is 15. Examples of the items for each skill area are: Communication: demonstrates understanding of the meaning of NO/YES; Daily Living: buttons clothes correctly without assistance; Socialization: expresses two or more recognizable emotions such as sadness, fear or distress and pleasure; Motor: runs smoothly with changes in speed and direction.

For the scale items the following reliability coefficients were obtained: Full scale Cronbach'alpha coefficient $=0.98$, Spearman brown coefficient $=0.95$, Guttman Split half coefficient $=0.94$; Subsets: Communication - Cronbach's alpha $=0.93$, Spearman Brown coeff. $=$ 0.84, Guttman Split half coeff. $=0.81$; Daily Living- Cronbach's alpha $=0.90$, Spearman Brown coeff. $=0.84$, Guttman Split half coeff. $=0.81$; Socialisation- Cronbach's alpha $=0.93$, Spearman Brown coeff. $=0.91$, Guttman Split half coeff. $=0.91 ;$ Motor - Cronbach's alpha $=0.94$, Spearman Brown coeff. $=0.86$, Guttman Split half coeff. $=0.86$.

\section{Procedure}

The children were rated by their teachers in terms of their competence levels in the four areas of adaptive behaviour skills studied.

\section{Results}

Means scores and standard deviations with respect to age and ethnic groups are presented in tables $1 \mathrm{a}$ and $1 \mathrm{~b}$, and with respect to age and gender in tables $2 \mathrm{a}$ and $2 \mathrm{~b}$.

Table 1a: Means and standard Deviation of Children's scores on Adaptive Behaviour scale as a function of age and ethnic Groups

\begin{tabular}{|c|c|c|c|c|c|c|c|c|}
\hline \multirow{3}{*}{$\begin{array}{l}\text { Age } \\
\text { (Years) }\end{array}$} & \multicolumn{4}{|c|}{ Communication skill } & \multicolumn{4}{|c|}{ Daily Living skill } \\
\hline & Yoruba & Igbo & Hausa & Total & Yoruba & Igbo & Hausa & Total \\
\hline & $\mathrm{M} \quad(\mathrm{SD})$ & $\mathrm{M} \quad(\mathrm{SD})$ & $\mathrm{M} \quad(\mathrm{SD})$ & $\mathrm{M}$ (SD) & $\mathrm{M} \quad(\mathrm{SD})$ & (SD) & $\mathrm{M} \quad(\mathrm{SD})$ & $\mathrm{M} \quad(\mathrm{SD})$ \\
\hline 2 & $\begin{array}{l}23.1 \\
\mathrm{~N}=15\end{array}$ & $\begin{array}{l}20.3(4.1) \\
\mathrm{N}=8\end{array}$ & - & $\begin{array}{l}22.1 \\
\mathrm{~N}=23\end{array}$ & $23.5(3.2)$ & 19.1 & - & 22.0 \\
\hline 3 & $\begin{array}{l}30.8(5.0) \\
N=31\end{array}$ & $\begin{array}{l}30.4 \\
\mathrm{~N}=14\end{array}$ & $\begin{array}{l}31.0 \\
\mathrm{~N}=3\end{array}$ & $\begin{array}{l}30.7 \\
\mathrm{~N}=48\end{array}$ & $29.2(3.5)$ & $29.6(4.0)$ & $29.0(5.3)$ & $29.3(3.7)$ \\
\hline 4 & $\begin{array}{l}33.9(6.6) \\
N=22\end{array}$ & $\begin{array}{l}43.4(1.5) \\
\mathrm{N}=5\end{array}$ & $\begin{array}{l}38.5(6.4) \\
\mathrm{N}=2\end{array}$ & $\begin{array}{l}35.8(7.0) \\
\mathrm{N}=29\end{array}$ & $31.1(3.8)$ & $37.0(2.4)$ & $28.5(5.0)$ & $31.9(4.3)$ \\
\hline 5 & $\begin{array}{l}39.9 \\
\mathrm{~N}=11\end{array}$ & $\begin{array}{l}35.2(5.4) \\
\mathrm{N}=10\end{array}$ & $\begin{array}{l}43.5(0.7) \\
\mathrm{N}=2\end{array}$ & $\begin{array}{l}38.2(5.5) \\
\mathrm{N}=23\end{array}$ & $38.4(4.7)$ & $32.6(3.2)$ & $38.5(0.7)$ & $35.9(4.8)$ \\
\hline 6 & $\begin{array}{l}42.2(4.0) \\
\mathrm{N}=24\end{array}$ & $\begin{array}{l}37.9 \\
N=13\end{array}$ & . & $\begin{array}{l}40.7(5.9) \\
\mathrm{N}=37\end{array}$ & $40.2(4.5)$ & $38.2(4.8)$ & - & $39.5(4.6)$ \\
\hline 7 & $\begin{array}{l}42.7 \\
\mathrm{~N}=28\end{array}$ & $\begin{array}{l}41.9 \\
\mathrm{~N}=23\end{array}$ & $\begin{array}{l}38.5(1.9) \\
\mathrm{N}=4\end{array}$ & $\begin{array}{l}42.1 \\
\mathrm{~N}=55\end{array}$ & $41.2(3.5)$ & $41.9(3.4)$ & $40.3(2.5)$ & $41.4(3.4)$ \\
\hline 8 & $\begin{array}{l}41.6 \\
N=24\end{array}$ & $\begin{array}{l}41.3 \\
\mathrm{~N}=12\end{array}$ & - & $\begin{array}{l}41.5 \\
\mathrm{~N}=36\end{array}$ & $41.0(3.9)$ & $41.1(5.8)$ & - & $41.1(4.6)$ \\
\hline 9 & $\begin{array}{l}44.1 \\
\mathrm{~N}=23\end{array}$ & $\begin{array}{l}42.6 \\
\mathrm{~N}=8\end{array}$ & - & $\begin{array}{l}43.7 \\
\mathrm{~N}=31\end{array}$ & $40.9(2.3)$ & 41.1 & - & $40.9(2.3)$ \\
\hline Total & $\begin{array}{l}37.67(8.0) \\
N=178\end{array}$ & $\begin{array}{l}37.1 \\
\mathrm{~N}=93\end{array}$ & $\begin{array}{l}37.4(5.1) \\
\mathrm{N}=11\end{array}$ & $\begin{array}{l}37.5(8.0) \\
\mathrm{N}=282\end{array}$ & $36.0(7.1)$ & $36.1(7.9)$ & $34.7(6.5)$ & $36.0(7.4)$ \\
\hline
\end{tabular}


Table 1b: Means and Standard deviation of Children's scores on Adaptive behaviour scale as a function of age and ethnic Groups

\begin{tabular}{|c|c|c|c|c|c|c|c|c|}
\hline \multirow{3}{*}{$\begin{array}{l}\text { Age } \\
\text { (Years) }\end{array}$} & \multicolumn{4}{|c|}{ Socialization skill } & \multicolumn{4}{|c|}{ Motor Skill } \\
\hline & Yoruba & Igbo & Hausa & Total & Yoruba & Igbo & Hausa & Total \\
\hline & $\mathrm{M} \quad(\mathrm{SD})$ & $\mathrm{M} \quad(\mathrm{SD})$ & $\mathrm{M} \quad(\mathrm{SD})$ & $\mathrm{M} \quad(\mathrm{SD})$ & M (SD) & $\mathrm{M}(\mathrm{SD})$ & M (SD) & M (SD) \\
\hline 2 & $\begin{array}{l}26.7(6.3) \\
N=15\end{array}$ & $\begin{array}{l}26.5 \\
\mathrm{~N}=8\end{array}$ & - & $\begin{array}{l}26.6 \\
\mathrm{~N}=23\end{array}$ & $22.1(3.7)$ & $19.8(3.3)$ & - & $21.3(3.7)$ \\
\hline 3 & $\begin{array}{l}35.0(5.9) \\
N=31\end{array}$ & $\begin{array}{l}35.1 \\
\mathrm{~N}=14\end{array}$ & $\begin{array}{l}36.3 \\
\mathrm{~N}=3\end{array}$ & $\begin{array}{l}35.1 \\
\mathrm{~N}=48\end{array}$ & $30.4(4.1)$ & $30.3(4.6)$ & $26.7(5.5)$ & $30.1(4.3)$ \\
\hline 4 & $\begin{array}{l}35.1 \\
\mathrm{~N}=22\end{array}$ & $\begin{array}{l}44.6(0.9) \\
\mathrm{N}=5\end{array}$ & $\begin{array}{l}32.5 \\
\mathrm{~N}=2\end{array}$ & $\begin{array}{l}36.5 \\
\mathrm{~N}=29\end{array}$ & $35.9(5.5)$ & $43.6(2.2)$ & $35.5(0.7)$ & $37.2(5.7)$ \\
\hline 5 & $\begin{array}{l}40.5 \\
\mathrm{~N}=11\end{array}$ & $\begin{array}{l}35.4(6.5) \\
N=10\end{array}$ & $\begin{array}{l}44.5(0.7) \\
\mathrm{N}=2\end{array}$ & $\begin{array}{l}38.6 \\
\mathrm{~N}=23\end{array}$ & $39.6(5.3)$ & $37.5(4.7)$ & $44.5(0.7)$ & $39.1(5.1)$ \\
\hline 6 & $\begin{array}{l}42.8 \\
\mathrm{~N}=24\end{array}$ & $\begin{array}{l}38.9 \\
N=13.6)\end{array}$ & - & $\begin{array}{l}41.4(4.5) \\
\mathrm{N}=27\end{array}$ & $41.6(5.4)$ & $37.5(7.7)$ & - & $40.2(6.5)$ \\
\hline 7 & $\begin{array}{l}41.9(3.7) \\
\mathrm{N}=28\end{array}$ & $\begin{array}{l}42.4(3.9) \\
\mathrm{N}=23\end{array}$ & $\begin{array}{l}38.3(3.0) \\
\mathrm{N}=4\end{array}$ & $\begin{array}{l}41.8(3.8) \\
\mathrm{N}=55\end{array}$ & $40.3(5.3)$ & $41.9(4.7)$ & $37.5(5.0)$ & $40.7(5.1)$ \\
\hline 8 & $\begin{array}{l}42.3 \\
\mathrm{~N}=24\end{array}$ & $\begin{array}{l}40.3 \\
\mathrm{~N}=12\end{array}$ & - & $\begin{array}{l}41.6(5.0) \\
\mathrm{N}=36\end{array}$ & $39.5(6.4)$ & $38.3(8.2)$ & - & $39.1(7.0)$ \\
\hline 9 & $\begin{array}{l}42.5(6.0) \\
\mathrm{N}=23\end{array}$ & $\begin{array}{l}42.6(5.0) \\
\mathrm{N}=8\end{array}$ & - & $\begin{array}{l}42.5 \\
\mathrm{~N}=31\end{array}$ & $44.4(2.0)$ & $43.3(3.2)$ & - & $44.1(2.4)$ \\
\hline Total & $\begin{array}{l}38.7(7.2) \\
\mathrm{N}=178\end{array}$ & $\begin{array}{l}38.5(6.8) \\
\mathrm{N}=93\end{array}$ & $\begin{array}{l}37.8 \\
\mathrm{~N}=11\end{array}$ & $\begin{array}{l}38.6(7.0) \\
\mathrm{N}=282\end{array}$ & $37.0(7.9)$ & $36.9(8.5)$ & $35.5(7.4)$ & $36.9(8.1)$ \\
\hline
\end{tabular}

Table 2a: Means and Standard Deviation of Children's scores on Adaptive behaviour scale as a function of age and gende

\begin{tabular}{|c|c|c|c|c|c|c|}
\hline \multirow{3}{*}{$\begin{array}{l}\text { Age } \\
\text { (Years) }\end{array}$} & \multicolumn{3}{|c|}{ Communication Skill } & \multicolumn{3}{|c|}{ Daily Living Skill } \\
\hline & Male & Female & \multirow{2}{*}{$\begin{array}{l}\text { Total } \\
\text { M (SD) }\end{array}$} & \multirow{2}{*}{$\begin{array}{l}\text { Male } \\
\text { M (SD) }\end{array}$} & \multirow{2}{*}{$\begin{array}{l}\text { Female } \\
\text { M (SD) }\end{array}$} & \multirow{2}{*}{$\begin{array}{l}\text { Total } \\
\mathrm{M} \quad(\mathrm{SD})\end{array}$} \\
\hline & $\mathrm{M} \quad(\mathrm{SD})$ & M (SD) & & & & \\
\hline 2 & $23.6(4.9)$ & $21.1(4.0)$ & 22.1 (4.4) & $21.9(3.7)$ & 22.1 & $22.0(4.1)$ \\
\hline$N=23$ & $\mathrm{~N}=9$ & $N=14$ & $\mathrm{~N}=23$ & & & \\
\hline 3 & $30.0(4.7)$ & $31.5(3.6)$ & 30.7 (4.3) & $28.7(3.3)$ & $30.1(4.0)$ & $29.3(3.7)$ \\
\hline $\mathrm{N}=48$ & $N=27$ & $N=21$ & $\mathrm{~N}=48$ & & & \\
\hline 4 & $36.9(7.0)$ & $35.0(7.0)$ & $35.8(7.0)$ & $32.4(3.7)$ & $31.6(4.8)$ & $31.9(4.3)$ \\
\hline$N=29$ & $N=13$ & $N=16$ & $\mathrm{~N}=29$ & & & \\
\hline 5 & $37.0(6.7)$ & $39.1(4.4)$ & $38.2(5.5)$ & $35.8(5.6)$ & $35.9(4.4)$ & $35.9(4.8)$ \\
\hline$N=23$ & $\mathrm{~N}=10$ & $\mathrm{~N}=13$ & $\mathrm{~N}=23$ & & & \\
\hline 6 & $41.3(5.9)$ & $40.2(6.0)$ & $40.7(5.9)$ & $40.3(4.4)$ & $38.8(4.9)$ & $39.5(4.6)$ \\
\hline $\mathrm{N}=37$ & $\mathrm{~N}=18$ & $N=19$ & $N=37$ & & & \\
\hline 7 & $41.8(4.0)$ & $42.3(3.7)$ & $42.1(3.8)$ & $40.8(4.0)$ & $42.0(2.6)$ & $41.4(3.4)$ \\
\hline $\mathrm{N}=55$ & $\mathrm{~N}=27$ & $\mathrm{~N}=28$ & $\mathrm{~N}=55$ & & & \\
\hline 8 & $42.54 .3)$ & $40.6(6.5)$ & $41.5(5.6)$ & $40.7(4.4)$ & $41.4(4.8)$ & $41.1(4.6)$ \\
\hline$N=36$ & $N=17$ & $N=19$ & $N=36$ & & & \\
\hline 9 & $42.5(6.3)$ & $45.0(0.0)$ & $43.7(4.6)$ & $40.6(2.7)$ & $41.3(1.8)$ & $40.9(2.3)$ \\
\hline $\mathrm{N}=31$ & $\mathrm{~N}=16$ & $\mathrm{~N}=15$ & $\mathrm{~N}=31$ & & & \\
\hline Total & $37.6(7.9)$ & $37.4(8.2)$ & $37.5(8.0)$ & $35.9(7.2)$ & $36.1(7.5)$ & $36.0(7.4)$ \\
\hline & $\mathrm{N}=137$ & $\mathrm{~N}=145$ & $\mathrm{~N}=282$ & & & \\
\hline
\end{tabular}


Table 2b: Means and Standard Deviation of Children's scores on Adaptive behaviour scale as a function of age and gender

\begin{tabular}{|c|c|c|c|c|c|c|c|}
\hline \multirow{3}{*}{$\begin{array}{l}\text { Age } \\
\text { (Years) }\end{array}$} & \multicolumn{3}{|c|}{ Socialization Skill } & \multicolumn{3}{|c|}{ Motor Skill } & All Skills \\
\hline & Male & Female & Total & Male & Female & Total & Total \\
\hline & $\mathrm{M}(\mathrm{SD})$ & $\mathrm{M}(\mathrm{SD})$ & $\mathrm{M} \quad(\mathrm{SD})$ & $\mathrm{M}(\mathrm{SD})$ & $\mathrm{M}$ (SD) & $\mathrm{M}(\mathrm{SD})$ & (SD) \\
\hline 2 & $27.9(4.8)$ & $25.8(5.7)$ & $26.6 \quad(5.4)$ & 22.0 & $20.9(2.9)$ & $21.3(3.7)$ & $92.0(12.6)$ \\
\hline $\mathrm{N}=23$ & & & $\mathrm{~N}=23$ & & & & \\
\hline 3 & $34.9(6.0)$ & $35.5(4.5)$ & $35.1 \quad(5.3)$ & $29.9(4.5)$ & $30.4(4.1)$ & $30.1(4.3)$ & $125.2(13.7)$ \\
\hline $\mathrm{N}=48$ & & & $\mathrm{~N}=48$ & & & & \\
\hline 4 & $37.8(7.7)$ & $35.5(8.7)$ & $36.5 \quad(8.2)$ & $37.8(5.5)$ & $36.8(5.9)$ & $37.2(5.7)$ & $141.5(23.4)$ \\
\hline $\mathrm{N}=29$ & & & $\mathrm{~N}=29$ & & & & \\
\hline 5 & $37.0(7.1)$ & $39.9(5.2)$ & $38.6 \quad(6.1)$ & $37.5(5.8)$ & $40.3(4.3)$ & $39.1(5.1)$ & $151.7(18.8)$ \\
\hline $\mathrm{N}=23$ & & & $\mathrm{~N}=23$ & & & & \\
\hline 6 & $41.3(4.5)$ & $41.5(4.6)$ & $41.4 \quad(4.5)$ & $40.8(6.5)$ & $39.5(6.7)$ & $40.2(6.5)$ & $161.7(19.9)$ \\
\hline $\mathrm{N}=37$ & & & $\mathrm{~N}=27$ & & & & \\
\hline 7 & $41.5(4.0)$ & $42.2(3.6)$ & $41.8 \quad(3.8)$ & $41.7(4.6)$ & $39.8(5.5)$ & $40.7(5.1)$ & $166.0(13.1)$ \\
\hline $\mathrm{N}=55$ & & & $\mathrm{~N}=55$ & & & & \\
\hline 8 & $41.4(4.5)$ & $41.9(5.5)$ & $41.6 \quad(5.0)$ & $38.9(7.1)$ & $39.3(7.1)$ & $39.1(7.0)$ & $163.3(17.4)$ \\
\hline $\mathrm{N}=36$ & & & $\mathrm{~N}=36$ & & & & \\
\hline 9 & $40.2(7.3)$ & $45.0(0.0)$ & $42.5 \quad(5.7)$ & $43.3(3.2)$ & $44.9(0.3)$ & $44.1(2.4)$ & $171.3(12.1)$ \\
\hline $\mathrm{N}=31$ & & & $\mathrm{~N}=31$ & & & & \\
\hline Total & $38.4(6.7)$ & $38.9(7.3)$ & $38.6 \quad(7.0)$ & $37.1(7.9)$ & $36.7(8.2)$ & $36.9(8.1)$ & $149.0(28.1)$ \\
\hline & & & $\mathrm{N}=282$ & & & & \\
\hline
\end{tabular}

Out of eight (8) age groups and three ethnic groups studied, Yoruba ethnic group scored higher than others in 5 age groups under communication skill domain (e.g. 2,6,7,8, \& 9), Igbo group scored higher in one age group (4), Hausa group scored higher in 2 age groups $(3,5)$. Igbo ethnic group scored higher than other groups in 5 age groups $(3,4,7,8,9)$, Yoruba group scored higher in 2 age groups $(2,6)$ while Hausa group scored higher in 1 age group (5) under daily living skill domain (table 1a). Under socialization skill domain Yoruba group scored higher in 3 age groups $(2,6,8)$, Igbo group scored higher in 3 age groups $(4,7,9)$ and Hausa group scored higher in 2 age groups $(3,5)$. For motor skill Yoruba group scored higher in 5 age groups $(2,3,6,8,9)$, Igbo group scored higher in 2 age groups $(4,7)$, Hausa group scored higher in one age group (5), (table $1 \mathrm{~b})$.

Females scored higher than males in all skills at ages 3, 5, and 9. They scored higher than males at age 7 in all skills except motor skill and at age 8 except communication skill. Males scored higher than females in all skills at age 4 , scored higher than females in all skills at age 2 except daily living and at age 6 except socialization (table 2a, 2b). In all, adaptive behaviour skills competence increased with age with a drop at age 8 (table $2 \mathrm{~b}$ ).

A MANOVA was conducted to examine the effect of age, ethnic group, and gender and their interactions on the adaptive behaviour skill variables. In addition follow up univariate and multiple comparison analyses were carried out and the outcome are presented in tables 3,4 , \& 5. 
Kinsola, E.F. \& Goma, R. (2016). Ethnic and Influences on Adaptive Behaviour of Some Nigerian Children. Advances in Social Sciences Research Journal, 3(13) 224-233.

Table 3: Multivariate Tests for adaptive behaviour scores

\begin{tabular}{llllll}
\hline \multirow{2}{*}{ Age } & Effect & Value & F & Hypothesis df & Error df \\
Age x Ethnicity & Wilks' Lambda & 0.22 & $16.2^{* *}$ & 28 & 866 \\
& Wilks' Lambda & 0.74 & $1.9^{*}$ & 40 & 911 \\
\hline
\end{tabular}

Note: ${ }^{*} \mathbf{p}<.05,{ }^{* *} \mathrm{p}<.01$

Table 4: Tests of Between-Subject Effects for Children's adaptive behaviour scores

\begin{tabular}{|c|c|c|c|c|c|}
\hline Source & $\begin{array}{l}\text { Dependent } \\
\text { Variable }\end{array}$ & $\begin{array}{l}\text { Type III } \\
\text { Sums of Squares }\end{array}$ & $\mathrm{df}$ & Mean Square & $\mathrm{F}$ \\
\hline \multirow[t]{4}{*}{ AGE } & Communication & 7401.61 & 7 & 1057.37 & $44.60 * *$ \\
\hline & Daily Living & 7738.65 & 7 & 1105.52 & $76.31 * *$ \\
\hline & Socialisation & 3652.30 & 7 & 521.76 & $19.11^{* *}$ \\
\hline & Motor & 7813.74 & 7 & 1116.25 & $41.85^{* *}$ \\
\hline $\begin{array}{l}\text { GENDER* } \\
\text { AGE }\end{array}$ & Communication & 344.73 & 7 & 49.25 & $2.08^{*}$ \\
\hline \multirow{4}{*}{$\begin{array}{l}\text { ETHNICITY* } \\
\text { AGE }\end{array}$} & Communication & 857.95 & 10 & 85.80 & $3.62 * *$ \\
\hline & Daily Living & 454.17 & 10 & 45.42 & $3.14 * *$ \\
\hline & Socialisation & 809.55 & 10 & 80.95 & $2.97 * *$ \\
\hline & Motor & 674.05 & 10 & 67.41 & $2.53 * *$ \\
\hline
\end{tabular}

Note: ${ }^{*} \mathbf{p}<.05,{ }^{* *} \mathrm{p}<.01$

Table 5: Multiple Comparison test for Children's Adaptive Behaviour scores as a function of Age

\begin{tabular}{ll}
\hline Age (Years) & Mean Differ \\
$2 \& 3$ & $-33.23^{* *}$ \\
$2 \& 4$ & $-49.48^{* *}$ \\
$2 \& 5$ & $-59.74^{* *}$ \\
$2 \& 6$ & $-69.78^{* *}$ \\
$2 \& 7$ & $-74.02 * *$ \\
$2 \& 8$ & $-71.31^{* *}$ \\
$2 \& 9$ & $-79.26^{* *}$ \\
$3 \& 4$ & $-16.25^{* *}$ \\
$3 \& 5$ & $-26.51^{* *}$ \\
$3 \& 6$ & $-36.55^{* *}$ \\
$3 \& 7$ & $-40.79^{* *}$ \\
$3 \& 8$ & $-38.08^{* *}$ \\
$3 \& 9$ & $-46.03^{* *}$ \\
$4 \& 6$ & $-20.30^{* *}$ \\
$4 \& 7$ & $-24.54 * *$ \\
$4 \& 8$ & $-21.82 * *$ \\
$4 \& 9$ & $-29.78^{* *}$ \\
$5 \& 9$ & $-19.52^{* *}$
\end{tabular}

Note: $* * \mathbf{p}<.01$ 
Results in table 3 indicated significant multivariate effects of Age, $F(28,866)=16.2$ Wilks' $\chi$ $=.22, \mathrm{p}<.01$, and significant Age $\mathrm{x}$ Ethnicity interaction, $\mathrm{F}(40,911)=1.9$ Wilks' $\chi=.74 \mathrm{p}<.05$

Follow up univariate analyses in table 4 indicated significant main effect for Age for all adaptive behaviour skills, significant Age x Gender interaction effect for communication skill and significant Age x Ethnicity interaction effect for all adaptive behaviour skills. For main effect of age the results are: Communication, $F(7,243)=44.60, p<.01$; Daily living, $F(7,243)$ $=76.31$, $\mathrm{p}<.01$; Socialization, $\mathrm{F}(7,243)=19.11$; Motor, $\mathrm{F}(7,243)=41.85$, $\mathrm{p}<.01$. Gender by Age interaction effect results are Communication: $F(7,243)=2.08, p<.05$. Ethnicity by age interaction effect results are: Communication, $\mathrm{F}(10,243)=3.62$, $\mathrm{p}<.01$; Daily living, $\mathrm{F}(10,243)$ $=3.14$, p<.01; Socialization, $F(10,243)=2.97$, p<.01; Motor, $F(10,243)=2.53, p<.01$, (table 4). Results in table 5 indicated pairs of age groups' mean scores that are significantly different at $\mathrm{p}$ $<.01$. These results offer partial support for the hypotheses formulated.

\section{DISCUSSION:}

The results of this study confirmed the predicted developmental trend in acquisition of adaptive behaviour skill competence. This was demonstrated by the significant multivariate effect for age

(table 3), and significant compared mean score differences of age group pairs (table 5). Furthermore the results also partially confirmed ethnic and gender contributions to adaptive behaviour skill competence. This was made evident in (table 3) by the significant multivariate effect for ethnicity $\mathrm{x}$ age interaction, and in (table 4) by the significant ethnicity $\mathrm{x}$ age interaction and gender $x$ age interaction. Specifically at age 4 Igbo ethnic group reported higher competence levels in all the skill domains when compared to other groups; at age 5 Hausa group recorded higher competence levels in all the skill domains when compared to other groups; while at ages 2 and 6 Yoruba group demonstrated higher competence in all the skill domains when compared to other groups. For ages $(3,7,8, \& 9)$ competence levels were shared among the ethnic groups. For example, for age 3, Hausa group reported higher competence in communication; Igbo group in daily living; Hausa group in socialisation; and Yoruba group in Motor. For age 7, Yoruba group demonstrated higher competence in communication, while Igbo group reported higher competence in daily living, socialization and motor skill domains. For age 8, Yoruba group reported higher competence in communication, socialization, and motor skills while Igbo group reported higher competence in daily living skill. For age 9, higher competence levels were reported by Yoruba group in communication and motor skills, while higher competence levels were reported by Igbo group in daily living and socialization skills. Although some of these mean differences appear marginal but the spread of the results across ages and ethnic groups is what may account for the significant ethnicity $\mathrm{x}$ age interaction. For gender $\mathrm{x}$ age interaction, it was only in communication that the interaction was significant. In this domain males reported higher competence at ages $(2,4,6, \& 8)$, while females reported higher competence at ages $(3,5,7,79)$. For other skill domains competence levels in them were also shared among male and females but the interactions in these cases were not significant because of the very marginal mean differences. Of note is the observation that the main effect for ethnicity and gender were not significant. Explanation for this seems to lie in the uneven sample sizes for ethnic groups (e.g. Yoruba, 178; Igbo, 93; Hausa, 11), as well as uneven cell sizes for age groups. Actually the Hausa ethnic group was not represented at ages $(2,6,8, \& 9)$. At age group levels where they were represented the cell numbers were very few, e.g. (ages 3, (3); 4 (2); 5 (2); 7 (4), (table 1a). However regardless of this limitation, the significant ethnicity $\mathrm{x}$ age interaction confirms an influencing role of ethnicity in adaptive 
behaviour skill competence and which indicated that at particular age group levels ethnicity contributes to competence in different adaptive behaviour skill domains. Variations in skill domain competence according to ethnic groups might have reflected areas of emphasis in adaptive behaviour skill development by parents in their child rearing practices approach. This ethnic contribution to adaptive behaviour development and competence agrees with past reports on ethnic differences in the school version of adaptive behaviour [e.g. [8, 4]].

The sample sizes for gender were more comparable and cell sizes for age under gender are closer. The competence levels of the skills were shared by males and females but the interactions were not significant except for communication domain. At particular age group levels in the communication domain females reported better competence (e.g. at ages 3, 5, 7, \&9), while males reported better competence at ages $(2,4,6, \& 8)$. The contribution of gender to adaptive behaviour skill competence in this research aligns with past findings about the contributory relationship between gender and adaptive behaviour skills [e.g. 6, 7, 8, 4].

In addition, the high reliability coefficients obtained for the total scale and the sub domains indicated that the scale is culturally appropriate and test items are appropriate for the sample studied. Finally, despite the limitations presented by the unbalanced representativeness of the study sample this research has contributed significantly to our understanding of the development of adaptive behaviour skills in children and the significant roles that ethnicity and gender can collectively or differentially play in the development and demonstration of adaptive behaviour skill competence by Nigerian children. It was therefore concluded that further research in which the sample size anomalies are rectified and the sizes increased to make them representative and even could reveal significant main effects for ethnicity and gender with respect to adaptive behaviour skill development and competence in Nigerian children.

\section{References}

1. Akinsola, E.F. (2011). "Omoluwabi's approach" to educating the African child. In A. Bame Nsamenang, \& Therese M.S. Tchombe (Eds.). Handbook of African Educational Theories and Practices: A generative Teacher Education Curriculum. Pp. 221-232.

2. American Association on Mental Retardation (AAMR) (2002). Mental retardation: Definition, Classification, and Systems of Support, 10th edition, Washington, DC: AAMR.

3. Goma, R.O., \& Akinsola, E.F. (2013) Adaptive Behaviour Assessment Scale (Culture-Sensitive)

4. Huberty, Thomas J. (1987). Factor analysis of the WISC-R and the Adaptive Behaviour Scale -School edition for a referral sample. Journal of School Psychology, 25, 405-410.

5. Keith, TZ, Harrison, PL, \& Ehly, SW. Effects of adaptive behaviour on achievement: Path analysis of a national sample. Professional School Psychology. 1987, 2, 205-215

6. Keller, H.R., (1986). In-school adaptive behaviour: Assessment domains of behaviour rating scales and child characteristics. Journal of Psycho-educational Assessment, 4, 1-12.

7. Keller, H.R. (1988). Children's adaptive behaviours: Measure and source generalizability. Journal of Psycho-educational Assessment, 6, 371-389.

8. Lambert, N.M., (1986). Evidence on age and ethnic status bias in factor scores and the comparison score for the AAMD adaptive Behaviour Scale - School Edition. Journal of School Psychology, 24, 143-153.

9. Masten, AS, \& Coatsworth, JD. (1998). The development of competence in favourable and unfavourable environments: Lessons from research on successful children. American Psychologist. 53, 205-220

10. Oakland, Thomas \& Harrison, Patti L. (Eds.). Adaptive Behaviour Assessment System - II: Clinical Use and Interpretation. (2008). USA: Academic Press 
11. Sparrow, SS, Balla, DA, \& Cicchetti, DV. Vineland adaptive behaviour scales. American Guidance Service; Circle Pines, MN: 1984.

12. Sparrow, S., Cicchetti, D., \& Balla, D. (2005). Vineland Adaptive Behaviour Scales, 2nd edition, Circle Pines, MN: American Guidance Service Publishing.

13. Taverna, L., Bornstein, Marc H., Putnick, Diane L., \& Axia, Giovanna (2011). Adaptive behaviours in young children: A unique cultural comparison in Italy. Journal of Cross Cultural Psychology, 42, (3), 445-465. Doi: 10.1177/0022022110362748 Retrieved December 23, 2013 from: jcc.sagepub.com/content/42/3/445 